\title{
Attractors for Multivalued Impulsive Systems: Existence and Applications to Reaction-Diffusion System
}

\author{
S. Dashkovskiy $\mathbb{D}^{1},{ }^{1}$ O. A. Kapustian, ${ }^{2}$ O. V. Kapustyan, ${ }^{2}$ and N. V. Gorban ${ }^{3}$ \\ ${ }^{1}$ University of Würzburg, Emil-Fischer-Str. 40, Würzburg 97074, Germany \\ ${ }^{2}$ Taras Shevchenko National University, Kyiv, Ukraine \\ ${ }^{3}$ National Technical University of Ukraine "Igor Sikorsky Kyiv Polytechnic Institute", Kyiv, Ukraine \\ Correspondence should be addressed to S. Dashkovskiy; sergey.dashkovskiy@uni-wuerzburg.de
}

Received 13 July 2021; Revised 11 October 2021; Accepted 16 October 2021; Published 5 November 2021

Academic Editor: zuowei cai

Copyright $\odot 2021$ S. Dashkovskiy et al. This is an open access article distributed under the Creative Commons Attribution License, which permits unrestricted use, distribution, and reproduction in any medium, provided the original work is properly cited.

In this paper, we develop a general approach to investigate limit dynamics of infinite-dimensional dissipative impulsive systems whose initial conditions do not uniquely determine their long time behavior. Based on the notion of an uniform attractor, we show how to describe limit behavior of such complex systems with the help of properties of their components. More precisely, we prove the existence of the uniform attractor for an impulsive multivalued system in terms of properties of nonimpulsive semiflow and impulsive parameters. We also give an application of these abstract results to the impulsive reaction-diffusion system without uniqueness.

\section{Introduction}

The global attractors' theory of dissipative dynamical systems provides information about the long-term behavior of the system which is rather valuable for the design of practical systems in the framework of mathematical systems and control theory. Such information is of interest for applications, but its derivation leads to challenges from the mathematical viewpoint problems, especially in the case of nonlinear infinite-dimensional systems. It is known that, in case of a finite dimensional phase space, a dissipative continuous dynamical system possesses a global attractor, that is, an invariant and globally, uniformly attracting compact set. To prove existence of such a set for an infinitedimensional continuous dynamical system, we have to impose additional assumption, asymptotic compactness, which can be verified in different ways for rather general classes of evolutionary processes [1-3]. Moreover, using the set-valued analysis approach [4], theory of global attractors can be applied to systems with nonunique solutions of the Cauchy problem [5-18].

However, in some practical situations, the dynamics is not always continuous due to effects such as elastic collisions, sampling of signals, triggered events, and other actions causing impulsive affects [19]. In mathematical modeling of the corresponding real-world applications in mechanics engineering, biology, etc., one has to deal with hybrid systems [20], in which discrete and continuous dynamics are combined in one model. An important subclass of such systems is impulsive dynamical systems. Their continuous dynamics is described by means of a continuous semigroup as long as the solution does not touch a given set in the state space. Having reached this set, the state changes instantaneously due to an impulsive action. Then, the dynamics is governed again by the continuous semigroup. Such systems were actively studied in view of their applications in mechanics [21-23]. Recently, this framework was used due to application in biology and medicine [19]. Qualitative analysis of such systems in finite dimensional case was carried out in [24-30].

The main problem we face when we try to expand the global attractor theory to impulsive DS is the lack of continuous dependence on the initial data. To overcome this difficulty, two approaches were proposed. One approach has been proposed and developed in [31-34]. The key idea of those papers is to keep the invariance property in the 
definition of the attractor. This approach allows to construct theory of attractors for impulsive DS similar to the classical one, but it requires additional information about impulsive trajectories in a neighborhood of the impulsive set and causes significant restrictions on their behavior.

Another approach was developed in [35-38] and uses the notion of the uniform attractor. An advantage of this approach is that we work with a compact uniformly attracting set without any restrictive assumptions on the impulsive semiflow. In the present paper, we develop this approach and prove the existence of the uniform attractor for the impulsive multivalued system in terms of properties of nonimpulsive semiflow and impulsive parameters. We also give an application of these abstract results to the impulsive reaction-diffusion system without uniqueness of solutions.

Let us note that this paper uses a common approach applied to study attracting sets of impulsive infinite-dimensional systems, as, for example, in [31-39]. In these and many other works, the existence of a uniform attractor is proved by means of asymptotic compactness verification for the impulsive semiflow. This verification is rather difficult already in case of relatively simple problems because the times of impulsive actions are usually not known in advance. The novelty of our results is that they provide new sufficient conditions for the asymptotic compactness property which can be verified easily (see Lemmas 2 and 3). This allows us to consider a more general class of systems with more general impulsive action classes. In particular, we were able to establish the existence of a uniform attractor for an impulsive system of $N \in \mathbb{N}$ reaction-diffusion equations in a general form.

\section{Setting of the Problem}

Assume that we have an evolution continuous system, i.e., the family $K$ of continuous maps $\varphi:[0,+\infty) \longrightarrow X$ :

(A1) $\forall x \in X \exists \varphi \in K$ such that $\varphi(0)=x$

(A2) $\forall \varphi \in K \forall \tau \geq 0 \varphi(\cdot+\tau) \in K$

In applications, the family $K$ is generated by autonomous evolution equation which is globally resolvable in the phase space $X$. Uniqueness of solution is not assumed.

Impulsive system $\{K, M, I\}$ is an evolution system such that a phase point $x(t)$ moves along trajectories of $K$ until it reaches a fixed subset $M \subset X$ which is called an impulsive set. At that moment $\tau$ (which is not fixed and depends on a particular trajectory), the phase point "jumps" into a new position $x^{+}(\tau) \in I x(\tau)$, where $I: M \longrightarrow X$ is called the impulsive map. After that, the phase point $x$ continues its motion along a trajectory of $K$ up to the next meeting with $M$. The trixial example is a bouncing ball described by phase vector $\left(\begin{array}{c}x \\ \dot{x}\end{array}\right)$, where "jumps" means instantaneous change of $\dot{x}$ when $\underset{x}{x}$ teaches a certain surface $M$. Under suitable assumptions (see Definition 1), such an impulsive system can be considered as a multivalued (discontinuous) dynamical system. If this system is dissipative, its limit behavior can be described by the investigation of properties of uniform attractor, minimal compact uniformly attracting set in the phase space. The main aim of the present paper is to give effective sufficient conditions for investigation of such sets in terms of $K, M$, and $I$. Under obtained conditions, we apply to study qualitative behavior of impulsive systems generated by reaction-diffusion evolution problems.

\section{Attracting Sets for Multivalued Impulsive Systems}

Let $(X,\|\cdot\|)$ be a Banach space; $P(X)(\beta(X))$ be a set of all nonempty (nonempty bounded) subsets of space $X$. Let $K$ be an evolution continuous system on $X$ :

$$
K_{x}=\{\varphi \in K: \varphi(0)=x\}
$$

Let $M \subset X$ be an impulsive set and $I: M \longrightarrow P(X)$ be an impulsive map. For $x \in M$, we denote by $x^{+}$some element of $I x$. For $\varphi \in K$, we denote $M^{+}(\varphi)=\cup \varphi(t) \cap M$. At the points of "jumps," we assume impulsive trajectories to be right continuous. For "well-posedness" of our impulsive problem, we assume the following conditions:

(A3) $M \subset X$ is closed and $I: M \longrightarrow P(X)$ is closedvalued

(A4) $M \cap I M=\varnothing$

(A5) $\forall x \in M \forall \varphi \in K_{x} \exists \tau=\tau(\varphi)>0$ such that $\forall t \in(0, \tau) \varphi(t) \notin M$

These assumptions allow us to claim [39] that, for every $\varphi \in K$, either $M^{+}(\varphi)=\varnothing$ or $\exists s=s(\varphi)>0$ such that $\varphi(s) \in M$ and $\varphi(t) \notin M \forall t \in(0, s)$. This function $s: K \longrightarrow(0,+\infty)$ (we put $s(\varphi)=+\infty$ if $M^{+}(\varphi)=\varnothing$ ) will play an important role in the sequel. In particular, it allows to define impulsive trajectory $\tilde{\varphi}$.

So, let $x_{0} \in X$ and $\varphi_{0} \in K_{x_{0}}$ be arbitrary. If $s\left(\varphi_{0}\right)=+\infty$, then $\widetilde{\varphi}(t)=\varphi_{0}(t) \forall t \geq 0$. Otherwise, we set $s_{0}=s\left(\varphi_{0}\right)$ and put

$$
\widetilde{\varphi}(t)= \begin{cases}\varphi_{0}(t), & t \in\left[0, s_{0}\right), \\ x_{1}^{+} \in I \varphi_{0}\left(s_{0}\right), & t=s_{0} .\end{cases}
$$

Let $\varphi_{1} \in K_{x_{1}^{+}}$. If $s\left(\varphi_{1}\right)=+\infty$, then $\tilde{\varphi}(t)=\varphi_{1}\left(t-s_{0}\right)$ $\forall t \geq s_{0}$. Otherwise, we set $s_{1}=s\left(\varphi_{1}\right)$ and put

$$
\tilde{\varphi}(t)= \begin{cases}\varphi_{1}\left(t-s_{0}\right), & t \in\left[s_{0}, s_{0}+s_{1}\right), \\ x_{2}^{+} \in I \varphi_{1}\left(s_{1}\right), & t=s_{0}+s_{1}\end{cases}
$$

Continuing this process, we obtain an impulsive trajectory $\widetilde{\varphi}$ with finite or infinite number of impulsive points $\left\{x_{n}^{+}\right\}_{n \geq 1} \subset I M$, corresponding time intervals between jumps $\left\{s_{n}\right\}_{n \geq 0}$, and functions $\left\{\varphi_{n}\right\}_{n \geq 0} \subset K$ such that $\forall n \geq 0$ and $\forall t \geq 0$ :

$$
\tilde{\varphi}(t)= \begin{cases}\varphi_{n}\left(t-t_{n}\right), & t \in\left[t_{n}, t_{n+1}\right), \\ x_{n+1}^{+} \in I \varphi_{n}\left(s_{n}\right), & t=t_{n+1},\end{cases}
$$

where $t_{0}=0$ and $t_{n+1}=\sum_{k=0}^{n} s_{k}$.

Denote by $\widetilde{K}\left(\widetilde{K}_{x}\right)$ the set of all (starting from $x$ ) impulsive trajectories. We assume the following condition.

(A6) $\forall x \in X$ and $\forall \widetilde{\varphi} \in \widetilde{K}_{x}: \widetilde{\varphi}$ is defined on $[0,+\infty)$, i.e., either the number of jumps is no more than finite (including nonimpulsive case) or $\sum_{n=0}^{\infty} s_{n}=\infty$ 
Remark 1. According to our construction, an impulsive trajectory may start from $M$ but cannot belong to $M$ at any positive time moment, i.e.,

$$
\forall x \in X \forall \widetilde{\varphi} \in \widetilde{K}_{x} \forall t>0: \widetilde{\varphi}(t) \notin M .
$$

Definition 1. A set-valued map $\widetilde{G}:[0,+\infty) \times X \longrightarrow P(X)$ given by

$$
\widetilde{G}(t, x)=\left\{\widetilde{\varphi}(t): \widetilde{\varphi} \in \widetilde{K}_{x}\right\}
$$

is called impulsive (or discontinuous) multivalued dynamical system.

It can be shown [38] that $\widetilde{G}$ is a multivalued semiflow (msemiflow), i.e.,

$$
\begin{array}{r}
\forall x \in X \widetilde{G}(0, x)=x, \\
\forall t, s \geq 0 \widetilde{G}(t+s, x) \subseteq \widetilde{G}(t, \widetilde{G}(s, x)) .
\end{array}
$$

In the last embedding, equality takes place if $\forall \varphi, \psi \in K$ such that $\varphi(0)=\psi(s)$; the concatenation function $\theta(p)=$ $\left\{\begin{array}{ll}\psi(p), & p \in[0, s) \\ \varphi(p-s), & p \geq s\end{array}\right.$ belongs to $K$.

Definition 2. A compact set $\Theta \subset X$ is called a uniform attractor of $\mathrm{m}$-semiflow $\widetilde{G}$ if $\Theta$ is a uniformly attracting set, i.e., $\forall B \in \beta(X)$ :

$$
\operatorname{dist}(\widetilde{G}(t, B), \Theta) \longrightarrow 0 \text { as } t \longrightarrow \infty \text {, }
$$

where $\Theta$ is the minimal set among all closed uniformly attracting sets.

Remark 2. In the classical dynamical systems theory (where $\widetilde{G}$ is single-valued and continuous map), the uniform attractor coincides with the global attractor, i.e., compact, invariant, and uniformly attracting set. As it was shown in [37], we cannot expect the invariance of the attractor in impulsive case.

The following lemma provides criteria for a dissipative semiflow to have a uniform attractor.

Lemma 1 (see [2]). Assume that $m$-semiflow $\widetilde{G}$ is dissipative, i.e., there exists $B_{0} \in \beta(X)$ such that, for every subset $B \in \beta(X)$ exists, the time moment $T=T(B)>0$ :

$$
\forall t \geq T(B) \widetilde{G}(t, B) \subset B_{0} .
$$

Then, $\widetilde{G}$ has a uniform attractor if and only if $\widetilde{G}$ is asymptotically compact, i.e., $\forall t_{n} \nearrow \infty$, and for all bounded sequences $\left\{x_{n}\right\} \in \beta(X)$, every sequence $\left\{\xi_{n}=\widetilde{G}\left(t_{n}, x_{n}\right)\right\}$ has a convergent subsequence.

In applications, dissipativity can be verified (only technical difficulties can appear) with the help of a priori estimates for trajectories of $K$. However, verification of asymptotically compactness for the infinite-dimensional impulsive problem is much more delicate problem. This property can be false in the simplest cases.

Example 1. Let us consider the following parabolic problem in bounded domain $\Omega \subset \mathbb{R}^{n}$ :

$$
\left\{\begin{array}{l}
\frac{\partial \varphi}{\partial t}=\Delta \varphi, \\
\left.\varphi\right|_{\partial \Omega}=0 .
\end{array}\right.
$$

In the phase space $X=L^{2}(\Omega)$ with $L^{2}$-norm $\|\cdot\|$, this problem is uniquely, globally resolvable and globally exponentially stable, i.e.,

$$
\forall t \geq 0\|\varphi(t)\| \leq e^{-\lambda_{1} t}\left\|\varphi_{0}\right\|,
$$

where $\lambda_{1}>0$ is the first eigenvalue of the operator $-\Delta$ in $H_{0}^{1}$.

Let us consider impulsive parameters:

$$
\begin{aligned}
M & =\{\varphi \in X:\|\varphi\|=\varepsilon\}, \varepsilon>0, \\
I: M \longrightarrow X, I \varphi & =(1+\mu) \varphi, \quad \mu>0 .
\end{aligned}
$$

It can be proved [35] that $\forall \varepsilon>0$ and $\forall \mu>0$ such that the impulsive system satisfies assumptions (A1)-(A6), and it is dissipative. However, it is not asymptotically compact. Therefore, it does not possess the uniform attractor.

The reason is that every trajectory meets the impulsive set $M$ too often, so the set of impulsive points is not compact.

For further consideration, we need additional assumptions which take place for the most evolution systems $K$.

(A7) For any sequence $\left\{x_{n}\right\}, x_{n} \longrightarrow x$ as $n \longrightarrow \infty$, and for any $\varphi_{n} \in K_{x_{n}}$, there exists $\varphi \in K_{x}$ such that, on some subsequence, $\varphi_{n}(t) \longrightarrow \varphi(t) \forall t \geq 0$

(A8) $\forall r>0 \exists c_{1}(r)$ such that $\forall x,\|x\| \leq r, \forall \varphi \in K_{x}$, and $\forall t \geq 0:\|\varphi(t)\| \leq c_{1}(r)$

Lemma 2. Assume that impulsive system $\{K, M, I\}$ satisfies conditions (A1)-(A8), the corresponding $m$-semiflow $\widetilde{G}$ is dissipative, and there exists compactly embedded space $Y \Subset X$ such that

(A9) $\forall t>0$ and $\forall r>0$ exist $c_{2}(t, r)$ such that $\forall x,\|x\| \leq r$ and $\forall \varphi \in K_{x}$; it is true that

$$
\|\varphi(t)\|_{Y} \leq c_{2}(t, r) .
$$

(A10) $\forall r>0 \exists c_{3}(r)$ such that $\forall x \in M \cap Y,\|x\|_{Y} \leq r$, and $\forall x^{+} \in I x$; it is true that

$$
\left\|x^{+}\right\|_{Y} \leq c_{3}(r) .
$$

(A11) $\exists \bar{s}>0$ such that $\forall x \in I(M \cap Y)$ and $\forall \varphi \in K_{x}$; it is true that $s(\varphi) \geq \bar{s}$.

Then, the impulsive m-semiflow $\widetilde{G}$ has a uniform attractor.

Remark 3. $Y \Subset X$ implies that $\exists \alpha>0$ such that $\forall x \in Y\|x\| \leq \alpha\|x\|_{Y}$, and if $\left\|x_{n}\right\|_{Y} \leq c$, then, up to subsequence, $x_{n} \longrightarrow x$ in $X$.

Remark 4. Assumption (A9) means that $K$ generates a compact semigroup. 
Proof. Dissipativity of m-semiflow $\widetilde{G}$ implies that $\forall r>0 \exists T(r)$ such that $\forall x,\|x\| \leq r, \forall \widetilde{\varphi} \in \widetilde{K}_{x}$, and $\forall t \geq T(r)$ : $\|\widetilde{\varphi}(t)\| \leq R_{0}$. Moreover, combining (A8), (A10), and (A11), we get that $\exists c(r)$ such that $\forall t \in[0, T(r)]\|\widetilde{\varphi}(t)\| \leq c(r)$. So, we can deduce that

$$
\begin{array}{r}
\forall r>0 \exists c(r) \text { such that } \forall x,\|x\| \leq r, \forall \widetilde{\varphi} \in \widetilde{K}_{x}, \forall t \geq 0 \\
\|\widetilde{\varphi}(t)\| \leq c(r)+R_{0}=: D(r) .
\end{array}
$$

Due to Lemma 1, we have to prove that the set,

$$
\left\{\xi_{m}=\tilde{\varphi}_{m}\left(t_{m}\right): \tilde{\varphi}_{m} \in \tilde{K}_{x_{m}},\left\|x_{m}\right\| \leq r, t_{m} \nearrow \infty\right\},
$$

is precompact in $X$.

So, we consider an impulsive trajectory $\widetilde{\varphi}_{m}$ which consists of impulsive points $\left\{x_{n}^{(m)+}\right\}_{n \geq 1} \subset I(M \cap Y)$, time intervals $\left\{s_{n}^{(m)}\right\}_{n \geq 0}$, and functions $\left\{\varphi_{n}^{(m)}\right\}_{n \geq 0} \subset K$. We have the following alternatives.

(a) If $t_{m}<s_{(0)}^{(m)}=s\left(\varphi_{(0)}^{(m)}\right) \leq \infty$, then

$$
\xi_{m}=\widetilde{\varphi}_{m}\left(t_{m}\right)=\varphi_{0}^{(m)}\left(t_{m}\right)=\varphi_{0}^{(m)}\left(1+t_{m}-1\right),
$$

$$
\varphi_{0}^{(m)}\left(\cdot+t_{m}-1\right) \in K_{\varphi_{0}^{(m)}\left(t_{m}-1\right)},
$$$$
\left\|\varphi_{0}^{(m)}\left(t_{m}-1\right)\right\| \leq D(r) .
$$

Hence, due to (A9), we have that

$$
\left\|\xi_{m}\right\|_{Y} \leq c_{2}(1, D(r)) .
$$

So, $\left\{\xi_{m}\right\}$ is precompact in $X$.

(b) If $t_{m}=s\left(\varphi_{0}^{(m)}\right)$, then $\xi_{m}=\widetilde{\varphi}_{m}\left(t_{m}\right) \in I \varphi_{0}^{(m)}\left(t_{m}\right)$. So, due to $(\mathrm{A} 10),\left\|\xi_{m}\right\|_{Y} \leq c_{3}\left(c_{2}(1, D(r))\right)$, and $\left\{\xi_{m}\right\}$ is precompact in $X$.

(c) If $t_{m}>s\left(\varphi_{0}^{(m)}\right)$, then, for some $n=n(m) \geq 1$,

$$
\xi_{m}=\widetilde{\varphi}_{m}\left(t_{m}\right)=\varphi_{n}^{(m)}\left(t_{m}-\sum_{k=0}^{m} s_{k}^{m}\right), \varphi_{n}^{(m)} \in K_{x_{n}^{(m)+}},
$$

or $\xi_{m}=x_{n+1}^{(m)+}$, and due to (15) and (A10), $\forall n \geq 1$,

$$
\left\|x_{n}^{(m)+}\right\|_{Y} \leq c_{3}(D(r)) \text {. }
$$

Let us denote $\tau_{m}=t_{m}-\sum_{k=0}^{m} s_{k}^{m}, \eta_{m}=x_{n}^{(m)+}$. Then, up to subsequence, $\eta_{m} \longrightarrow \eta$ in $X$.

If $\tau_{m} \longrightarrow \tau \geq 0$, then, due to (A7), for some $\varphi \in K_{\eta}$, up to subsequence,

$$
\varphi_{n}^{(m)}\left(\tau_{m}\right) \longrightarrow \varphi(\tau) \text { as } m \longrightarrow \infty .
$$

So, $\left\{\xi_{m}\right\}$ is precompact.

Otherwise, $\tau_{m} \longrightarrow \infty$. Then, arguing analogously as at to the beginning of the proof, we obtain

$$
\left\|\xi_{m}\right\|_{Y} \leq c_{2}(1, D(r)) \text {. }
$$

Lemma 3. Assume that impulsive system $\{K, M, I\}$ satisfies conditions (A1)-(A8), the corresponding $m$-semiflow $\widetilde{G}$ is dissipative, and there exists a compactly embedded space $Y \Subset X$.

$\left(A 9^{\prime}\right) \forall x \in X, \forall \varphi \in K_{x}$, and $\forall t \geq 0$,

$$
\varphi(t)=\varphi_{1}(t)+\varphi_{2}(t)
$$

where

$$
\left\|\varphi_{1}(t)\right\|_{Y} \leq c_{4}(\|x\|), c_{4}(\cdot) \text { is continuous on }[0,+\infty],
$$

$\left\|\varphi_{2}(t)\right\| \leq c_{5}\|x\| e^{-\delta t}$, where $\delta>0$, and $c_{5} \geq 0$ are constants.

$\left(A 10^{\prime}\right) \exists \bar{x} \in X: \forall r>0 \exists c_{6}(r)$ such that $\forall x \in M,\|x\| \leq r$, and $\forall x^{+} \in I x$; it is true that

$$
\left\|x^{+}-\bar{x}\right\|_{Y} \leq c_{6}(r) \text {. }
$$

$\left(A 11^{\prime}\right) \exists \bar{s}>0$ such that $\forall x \in I M$ and $\forall \varphi \in K_{x}$; it is true that $s(\varphi) \geq \bar{s}$.

Then, the impulsive m-semiflow $\widetilde{G}$ has a uniform attractor.

Remark 5. Assumption $\left(\mathrm{A}^{\prime}\right)$ means that $K$ generates an asymptotically compact semigroup.

Proof. The alternatives (a)-(c) of the proof are the same as in the proof of Lemma 2. We consider an impulsive trajectory $\widetilde{\varphi}_{m}$ with components $\left\{x_{n}^{(m)+}\right\}_{n \geq 1} \subset I M,\left\{s_{n}^{(m)}\right\}_{n \geq 0}$, $\left\{\varphi_{n}^{(m)}\right\}_{n \geq 0} \subset K$, and $\left\|\widetilde{\varphi}_{m}(0)\right\| \leq r$, and (15) takes place.

(a) If $t_{m}<s_{0}^{(m)}=s\left(\varphi_{0}^{m}\right) \leq \infty$, then, due to (A9'),

$$
\xi_{m}=\widetilde{\varphi}_{m}\left(t_{m}\right)=\varphi_{0}^{(m)}\left(t_{m}\right)=\left(\varphi_{0}^{(m)}\right)_{1}\left(t_{m}\right)+\left(\varphi_{0}^{(m)}\right)_{2}\left(t_{m}\right),
$$

where $\left\|\left(\varphi_{0}^{(m)}\right)_{1}\left(t_{m}\right)\right\|_{Y} \leq c_{4}(r)$ and $\left\|\left(\varphi_{0}^{(m)}\right)_{2}\left(t_{m}\right)\right\|$ $\leq c_{5} r e^{-\delta t_{m}}$.

So, the first summand has a convergent subsequence, and the second one tends to zero as $m \longrightarrow \infty$. Therefore, $\left\{\xi_{m}\right\}$ is precompact.

(b) If $t_{m}=s_{0}^{(m)}$, then

$$
\xi_{m}=\widetilde{\varphi}\left(t_{m}\right) \in I \varphi_{0}^{(m)}\left(t_{m}\right) .
$$

Due to case (a), we have that

$$
\left\|\varphi_{0}^{(m)}\left(t_{m}\right)\right\| \leq \alpha c_{4}(r)+c_{5} r .
$$

So, from (A11'),

$$
\left\|\xi_{m}-\bar{x}\right\|_{Y} \leq c_{6}\left(\alpha c_{4}(r)+c_{5} r\right) .
$$

It means precompactness of $\left\{\xi_{n}\right\}$ in $X$.

(c) If $t_{m}>s\left(\varphi_{0}^{(m)}\right)$, then, for some $n=n(m) \geq 1$,

$$
\xi_{m}=\tilde{\varphi}\left(t_{m}\right)=\varphi_{n}^{(m)}\left(\tau_{m}\right), \varphi_{n}^{(m)} \in K_{\eta_{m}},
$$

where $\tau_{m}=t_{m}-\sum_{k=0}^{m} s_{k}^{(m)}$ and $\eta_{m}=x_{n}^{(m)+}$. 
Due to (A10'),

$$
\left\|\eta_{m}-\bar{x}\right\|_{Y} \leq c_{6}(D(r)) .
$$

So, up to subsequence, $\eta_{m} \longrightarrow \eta$ in $X$. After that, we can repeat arguments from Lemma 2 and finish the proof.

\section{Application to Reaction-Diffusion Impulsive System}

We consider the reaction-diffusion type system:

$$
\left\{\begin{array}{l}
\frac{\partial u}{\partial t}=A \Delta u+\varepsilon f(u), \quad t>0, x \in \Omega \\
\left.u\right|_{\partial \Omega}=0
\end{array}\right.
$$

where $\Omega \subset \mathbb{R}^{d}$ is a bounded domain, $\varepsilon>0$ is a small parameter, $u=\left(u_{1}, \ldots, u_{N}\right)^{T}$ is unknown vector-function, $A$ is $N \times N$ matrix with positive symmetric part $A+A^{T} / 2 \geq \beta I, \beta>0, \quad f=\left(f_{1}, \ldots, f_{N}\right)^{T}$ is continuous nonlinear interaction vector-function, and

$$
\sup _{u \in \mathbb{R}^{N}}\|f(u)\|_{\mathbb{R}^{N}} \leq C
$$

It is known [2] that, under such assumptions, for every $u(0) \in X=\left(L^{2}(\Omega)\right)^{N}$, there exists (maybe nonunique) global weak solution of problem (32), and every weak solution belongs to $\mathbb{C}([0,+\infty), X)$.

We consider the following impulsive problem:

$$
M=\left\{u \in X: \sum_{i=1}^{N}\left(u_{i}, \psi\right)^{2}=1\right\},
$$

where $\psi$ is chosen as one of solutions of the spectral problem:

$$
\Delta \psi=-\lambda \psi, \psi \in H_{0}^{1}(\Omega) .
$$

We put $L=\left\{\bar{c} \psi: \bar{c} \in \mathbb{R}^{N}\right\}$. Then, $X=L \oplus L^{\perp}$, and we put, for $u \in M, u=\bar{c} \psi+u^{\perp}, u^{\perp} \in L^{\perp}, \sum_{i=1}^{N} \bar{c}_{i}^{2}=1$, and

$$
I(u)=\left\{v=\bar{d} \psi+u^{\perp}: \sum_{i=1}^{N} \bar{d}_{i}^{2}=1+\mu\right\} .
$$

Remark 6. In the case, $N=1$, the described impulsive problem consists of increasing $1+\mu$ times the value $(u, \psi)^{2}$.

Let $K$ be the set of global weak solutions of problem (32).

Theorem 1. The impulsive system $\{K, M, I\}$ has the uniform attractor in the phase space $X$.

Proof. Let us verify assumptions from Lemma 2. Within this proof constant, $C$ means a constant which depends on parameters of (32).

It is known that $K$ satisfies (A1), (A2), (A7), and (A8). Moreover, for every $u \in K$,

$$
\forall t \geq 0\|u(t)\|_{X}^{2} \leq\|u(0)\|_{X}^{2} e^{-\beta t}+1 .
$$

Additionally, for $Y=\left(H_{0}^{1}(\Omega)\right)^{N} \Subset X$, assumption (A9) takes place. Let us take $u \in M \cap Y$. Then,

$$
\|u\|_{Y}^{2}=\|\bar{c} \psi\|_{Y}^{2}+\left\|u^{\perp}\right\|_{Y}^{2}
$$

And, for arbitrary $u^{+} \in I(u)$,

$$
\left\|u^{+}\right\|_{Y}^{2}=\|\bar{d} \psi\|_{Y}^{2}+\left\|u^{\perp}\right\|_{Y}^{2} .
$$

So, if $\|u\|_{Y}^{2} \leq r^{2}$, then

$$
\left\|u^{+}\right\|_{Y}^{2} \leq r^{2}+\|\psi\|_{Y}^{2}\left(\sum_{i=1}^{N} \bar{d}_{i}^{2}-\sum_{i=1}^{N} \bar{c}_{i}^{2}\right) \leq r^{2}+\mu\|\psi\|_{Y}^{2} .
$$

Hence, (A10) takes place.

Also, it is easy to see that $M \subset X$ is closed, $\forall u \in X I(u)$ is closed, and $M \cap I M=\varnothing$. So, (A3) and (A4) hold.

For verifying other assumptions, we need more detailed analysis of our system. Let us introduce denotations:

$$
\begin{gathered}
\bar{v}(t)=\left(\begin{array}{c}
\left(u_{1}(t), \psi\right) \\
\ldots \\
9 u_{N}(t), \psi
\end{array}\right), \\
\bar{f}(u(t))=\left(\begin{array}{c}
\left(f_{1}(u), \psi\right) \\
\ldots \\
\left(f_{N}(u), \psi\right)
\end{array}\right) .
\end{gathered}
$$

Then, from (32), we obtain

$$
\frac{\mathrm{d}}{\mathrm{d} t} \bar{v}(t)+\lambda A \bar{v}(t)=\varepsilon \bar{f}(u(t))
$$

And, after multiplying by $\bar{v}(t)$ in $\mathbb{R}^{N}$, we obtain $\frac{1}{2} \frac{\mathrm{d}}{\mathrm{d} t}\|\bar{v}(t)\|_{\mathbb{R}^{N}}^{2}+\lambda(A \bar{v}(t), \bar{v}(t))_{\mathbb{R}^{N}}=\varepsilon(\bar{f}(u(t)), \bar{v}(t))_{\mathbb{R}^{N}}$.

Hence,

$\frac{\mathrm{d}}{\mathrm{d} t}\|\bar{v}(t)\|_{\mathbb{R}^{N}}^{2}+\lambda\left(\left(A+A^{T}\right) \bar{v}(t), \bar{v}(t)\right)_{\mathbb{R}^{N}}=2 \varepsilon(\bar{f}(u(t)), \bar{v}(t))_{\mathbb{R}^{N}}$.

So, $\forall t \geq 0$ :

$$
\sum_{i=1}^{N}\left(u_{i}(t), \psi\right)^{2} \leq \sum_{i=1}^{N}\left(u_{i}(0), \psi\right)^{2} e^{-\beta \lambda t}+\varepsilon^{2} C .
$$

Now, let us consider

$$
g_{\varepsilon}(t)=\|\bar{v}(t)\|_{\mathbb{R}^{N}}^{2}=\sum_{i=1}^{N}\left(u_{i}(t), \psi\right)^{2} .
$$

Here, index $\varepsilon$ means that $u(t)=\left(u_{1}(t), \ldots, u_{N}(t)\right)^{T}$ is a solution of problem (32) with $\varepsilon>0$.

Assume that $u_{0} \in M$. Then, $g_{\varepsilon}(0)=1$, and due to (44), we have that

$$
g_{\varepsilon}^{\prime}(0) \leq-2 \beta \lambda+2 \varepsilon C \text {. }
$$

Inequality (47) means that, for sufficiently small $\varepsilon>0$, it is true that $g_{\varepsilon}^{\prime}(0) \leq-\beta \lambda$. So, for such $\varepsilon>0 \exists \tau=\tau\left(u_{0}, \varepsilon\right)$ : $\forall t \in(0, \tau) g_{\varepsilon}(t)<1$. Hence, (A5) takes place. 
Now, let us take $u_{0} \in I M$. Assume that $s_{\varepsilon}>0$ is the first meeting of $M$ by $u \in K_{u_{0}}$. Then, $g_{\varepsilon}\left(s_{\varepsilon}\right)=1$, i.e., due to (44),

$$
\begin{aligned}
& 1+\lambda \int_{0}^{s_{\varepsilon}}\left(\left(A+A^{T}\right) \bar{v}(t), \bar{v}(t)\right)_{\mathbb{R}^{N}} \\
& =1+\mu+2 \varepsilon \int_{0}^{s_{\varepsilon}}(\bar{f}(u(t)), \bar{v}(t))_{\mathbb{R}^{N}} .
\end{aligned}
$$

Due to (45), $\forall t \in\left[0, s_{\varepsilon}\right]$ :

$$
\|\bar{v}(t)\|_{\mathbb{R}^{N}}^{2} \leq 1+\mu+\varepsilon^{2} C \leq 2+\mu,
$$

for sufficiently small $\varepsilon$.

So,

$$
\left|\left(\left(A+A^{T}\right) \bar{v}(t), \bar{v}(t)\right)_{\mathbb{R}^{N}}\right| \leq 2\|A\|(2+\mu)
$$

and

$$
2 \lambda\|A\|(2+\mu) s_{\varepsilon} \geq \mu-2 \varepsilon C \sqrt{2+\mu} s_{\varepsilon} .
$$

Finally,

$$
s_{\varepsilon} \geq \frac{\mu}{2 \lambda\|A\|(2+\mu)+2 C \sqrt{2+\mu}}=\bar{s} .
$$

Therefore, (A11) holds.

It is left to prove dissipativity property. Let us consider $\widetilde{\varphi} \in \widetilde{K}_{u_{0}}$ with $\left\|u_{0}\right\| \leq r$.

If $\tilde{\varphi}$ has not impulsive perturbation, then, from (37),

$$
\forall t \geq T=\frac{2}{\beta} \ln r:\|\widetilde{\varphi}(t)\|_{X} \leq \sqrt{2} .
$$

Otherwise, without loss of generality, we can assume that $u_{0} \in I M,\left\|u_{0}\right\| \leq r$, and $\widetilde{\varphi}$ has jumps at the moments $\left\{s_{0}, s_{0}+s_{1}, \ldots\right\}$ with impulsive points $\left\{y_{i}^{+}\right\}_{i \geq 1}$. Then, using (52) and equality $\left\|u^{+}\right\|=\|u\|^{2}+\mu \forall u \in M$, we have that, for $k \geq 0$,

$$
\left\|u_{k+1}^{+}\right\|^{2} \leq\left\|u_{0}\right\|^{2} e^{-\beta(k+1) \bar{s}}+(1+\mu) \sum_{i=0}^{k} e^{-\beta i \bar{s}} .
$$

Combining (53) and (54), we obtain required dissipativity. The theorem is proved.

\section{Data Availability}

No data were used to support this study

\section{Conflicts of Interest}

The authors declare that they have no conflicts of interest.

\section{Acknowledgments}

This work was supported by the German Research Foundation (DFG) (Grant no. DA 767/12-1) and the National Research Foundation of Ukraine (NRFU) (Grant no. NRFU F81/41743) through the joint German-Ukrainian project "Stability and robustness of attractors of nonlinear infinite-dimensional systems with respect to disturbances."

\section{References}

[1] R. Temam, Infinite-Dimensional Dynamical Systems in Mechanics and Physics, p. 500, Springer, New York, NY, USA, 1988.

[2] V. V. Chepyzhov and M. I. Vishik, Attractors of Equations of Mathematical Physics, AMS, Providence, RI, USA, 2002.

[3] J. C. Robinson, Infinite-dimensional Dynamical Systems, Cambridge University Press, Cambridge, UK, 2001.

[4] J.-P. Aubin and H. Frankowska, Set-valued Analisys, Birkhausser, Boston, MA, USA, 1990.

[5] J. M. Ball, "Continuity properties and global attractors of generalized semiflows and the navier-stokes equations," Journal of Nonlinear Science, vol. 7, no. 5, pp. 475-502, 1997.

[6] V. S. Melnik and J. Valero, "On attractors of multivalued semi-flows and differential inclusions," Set-Valued Analysis, vol. 6, pp. 83-111, 1998.

[7] A. V. Kapustyan, "Global attractors of a nonautonomous reaction-diffusion equation," Differential Equations, vol. 38, no. 10 , pp. 1467-1471, 2002.

[8] T. Caraballo, P. Marín-Rubio, and J. C. Robinson, "A comparison between two theories for multivalued semiflows and their asymptotic behavior," Set-Valued Analysis, vol. 11, no. 3, pp. 297-322, 2003.

[9] A. N. Carvalho and C. B. Gentile, "Asymptotic behaviour of non-linear parabolic equations with monotone principal part," Journal of Mathematical Analysis and Applications, vol. 280, no. 2, pp. 252-272, 2003.

[10] O. V. Kapustyan and D. V. Shkundin, "Global attractor of one nonlinear parabolic equation," Ukrainian Mathematical Journal, vol. 55, no. 4, pp. 446-455, 2003.

[11] J. M. Ball, "Global attractors for damped semilinear wave equations," Discrete and Continuous Dynamical Systems, vol. 10, pp. 31-52, 2004.

[12] P. E. Kloeden and J. Valero, "Attractors of weakly asymptotically compact set-valued dynamical systems," Set-Valued Analysis, vol. 13, no. 4, pp. 381-404, 2005.

[13] J. Simsen and C. Gentile, "On attractors for multivalued semigroups defined by generalized semiflows," Set-Valued Analysis, vol. 16, pp. 105-124, 2008.

[14] P. O. Kasyanov, "Multivalued dynamics of solutions of an autonomous differential-operator inclusion with pseudomonotone nonlinearity," Cybernetics and Systems Analysis, vol. 47, no. 5, pp. 800-811, 2011.

[15] P. Kalita and G. Lukaszewich, "Global attractors for multivalued semiflows with weak continuity properties," Nonlinear Analysis Series A, Theory, Methods and Applications, vol. 101, pp. 124-143, 2014.

[16] N. V. Gorban and P. O. Kasyanov, "On regularity of all weak solutions and their attractors for reaction-diffusion inclusion in unbounded domain," Continuous and Distributed Systems, vol. 211, pp. 205-220, 2014.

[17] N. V. Gorban, A. V. Kapustyan, E. A. Kapustyan, and O. V. Khomenko, "Strong global attractor for the three-dimensional Navier-Stokes system of equations in unbounded domain of channel type," Journal of Automation and Information Sciences, vol. 47, no. 11, pp. 48-59, 2015.

[18] O. V. Kapustyan, P. O. Kasyanov, and J. Valero, "Structure of the global attractor for weak solutions of a reaction-diffusion equation," Applied Mathematics and Information Sciences, vol. 9, no. 5, pp. 2257-2264, 2015.

[19] E. M. Izhkevich, Dynamical Systems in Neuroscience: The Geometry of Excitability and Bursting, The MIT Press, Cambridge, UK, 2007. 
[20] R. Goebel, R. Sanfelice, and A. R. Teel, Hybrid Dynamical Systems: Modeling, Stability, and Robustness, Princeton University Press, Princeton, NJ, USA, 2012.

[21] A. M. Samoilenko and N. A. Perestyuk, Impulsive Differential Equitations, World Scientific, Singapore, 1995.

[22] V. Lakshmikantham, D. D. Bainov, and P. S. Simeonov, Theory of Impulsive Differential Equitations, World Scientific, Singapore, 1989.

[23] M. Akhmet, Principles of Discontinuous Dynamical Systems, Springer, New York, NY, USA, 2010.

[24] K. Ciesielski, "On stability in impulsive dynamical systems," Bulletin of the Polish Academy of Sciences Mathematics, vol. 52, no. 1, pp. 81-91, 2004.

[25] E. M. Bonotto and M. Federson, "Limit sets and the PoincaréBendixson theorem in impulsive semidynamical systems," Journal of Differential Equations, vol. 244, no. 9, pp. 2334-2349, 2008.

[26] E. M. Bonotto, "Flows of characteristic 0+ in impulsive semidynamical systems," Journal of Mathematical Analysis and Applications, vol. 332, pp. 81-96, 2007.

[27] S. Dashkovskiy and M. Kosmykov, "Input-to-state stability of interconnected hybrid systems," Automatica, vol. 49, no. 4, pp. 1068-1074, 2013.

[28] S. Dashkovskiy and A. Mironchenko, "Input-to-state stability of nonlinear impulsive systems," SIAM Journal on Control and Optimization, vol. 51, no. 3, pp. 1962-1987, 2013.

[29] P. Feketa and Y. Perestyuk, "Perturbation theorems for a multifrequency system with pulses," Journal of Mathematical Sciences, vol. 217, no. 4, pp. 515-524, 2016.

[30] S. Dashkovskiy and P. Feketa, "Asymptotic properties of Zeno solutions," Nonlinear Analysis: Hybrid Systems, vol. 30, pp. 256-265, 2018.

[31] E. M. Bonotto and D. P. Demuner, "Attractors of impulsive dissipative semidynamical systems," Bulletin des Sciences Mathematiques, vol. 137, no. 5, pp. 617-642, 2013.

[32] E. M. Bonotto, M. C. Bortolan, A. N. Carvalho, and R. Czaja, "Global attractors for impulsive dynamical systems-a precompact approach," Journal of Differential Equations, vol. 259, no. 7, pp. 2602-2625, 2015.

[33] E. M. Bonotto, M. C. Bortolan, R. Collegary, and R. Czaja, "Semicontinuity of attractors for impulsive dynamical systems," Journal of Differential Equations, vol. 261, pp. 4358-4367, 2016.

[34] E. de Mello Bonotto and P. Kalita, "On attractors of generalized semiflows with impulses," Journal of Geometric Analysis, vol. 30, no. 2, pp. 1412-1449, 2019.

[35] M. O. Perestyuk and O. V. Kapustyan, "Global attractors of impulsive infinite-dimensional systems," Ukrainian Mathematical Journal, vol. 68, no. 4, pp. 517-528, 2016.

[36] S. Dashkovskiy, O. V. Kapustyan, and I. V. Romaniuk, "Global atractors of impulsive parabolic inclusions," Descrete and Continuous Dynamical Systems, Series B, vol. 22, no. 5, pp. 1875-1886, 2017.

[37] O. V. Kapustyan, M. O. Perestyuk, and I. V. Romanyuk, "Stability of global attractors of impulsive infinite-dimensional systems," Ukrainian Mathematical Journal, vol. 70, no. 1, pp. 30-41, 2018.

[38] S. Dashkovskiy, P. Feketa, O. Kapustyan, and I. Romaniuk, "Invariance and stability of global attractors for multi-valued impulsive dynamical systems," Journal of Mathematical Analysis and Applications, vol. 458, no. 1, pp. 193-218, 2018.

[39] S. Dashkovskiy, P. Feketa, O. V. Kapustyan, and I. V. Romaniuk, "Existence and invariance of global attractors for impulsive parabolic system without uniqueness," Understanding Complex Systems, Springer, New York, NY, USA, pp. 57-78, 2019. 